Document downloaded from:

http://hdl.handle.net/10251/50795

This paper must be cited as:

Reig, J.; Rubio Arjona, L.; Rodrigo Peñarrocha, VM. (2006). Performance of Dual Selection Combiners Over Correlated Nakagami-m Fading With Different Fading Parameters. IEEE Transactions on Communications. 54(9):1527-1532. doi:10.1109/TCOMM.2006.881188.

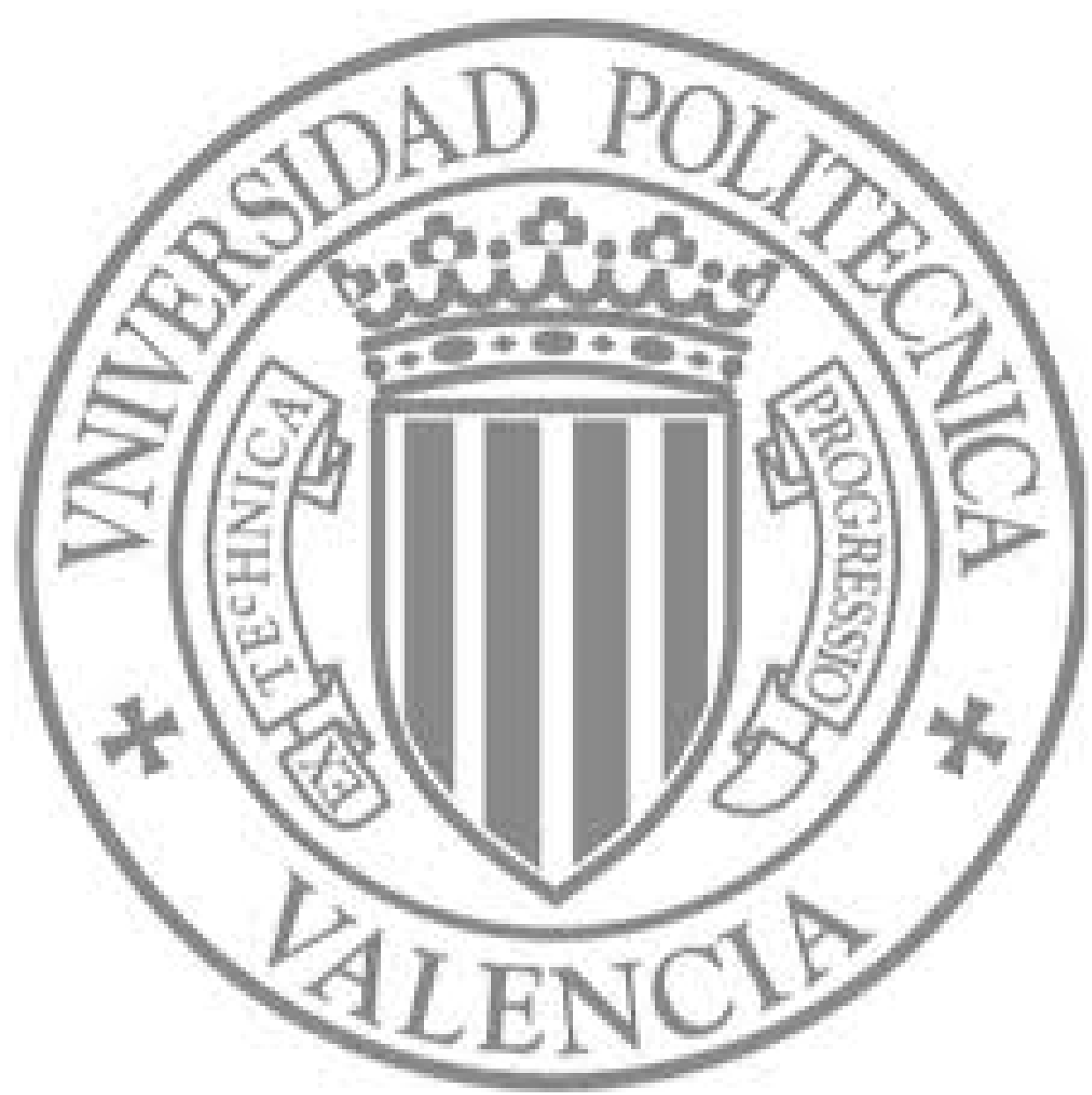

The final publication is available at

http://dx.doi.org/10.1109/TCOMM.2006.881188

Copyright Institute of Electrical and Electronics Engineers (IEEE) 


\title{
Performance of Dual Selection Combiners over Correlated Nakagami- $m$ Fading with Different Fading Parameters
}

${ }^{1}$ Juan Reig, Member, IEEE, Lorenzo Rubio, Member IEEE, and Vicent M. Rodrigo-Peñarrocha, Member IEEE

\begin{abstract}
This letter presents infinite series expressions for the outage probability, the probability density function (PDF), the average error probability for binary modulations, and average signal-to-noise ratio (SNR) of dual selection combiners (SC) over correlated fading with arbitrary fading parameters at each input of the combiner. The outage probability is calculated for both thermal noise and interference-limited scenarios. The results obtained for the outage probabilities specified for identical fading parameters at both branches of the combiner are contrasted with the results of other studies in the literature.
\end{abstract}

\section{Index terms}

Fading channels, correlation, land mobile radio diversity systems

\footnotetext{
${ }^{1}$ Dr. Reig, Dr. Rubio, and Dr. Rodrigo-Peñarrocha are with the Departamento de Comunicaciones, Universidad Politécnica de Valencia, 46022 Valencia, jreigp@dcom.upv.es, lrubio@dcom.upv.es This work was supported by the Research Support Program of the Generalitat Valenciana. Direcció General d'Investigació i Transferència Tecnològica. Ref.:GV04B/357.
} 


\section{INTRODUCTION}

Diversity techniques are used in wireless communications systems to reduce the effects of fading and to improve performance metrics, such as average signal-to-noise ratio (SNR), average probability of error, and outage probability. Among the most popular diversity techniques is selection combining (SC), which is noted for its simplicity. The performance of selection combiners over Nakagami- $m$ fading has been studied widely in the literature for identical fading parameters. Previously published works that are related to the evaluation of metrics in a noise-limited scenario, where the thermal interference power is negligible as contrasted with the thermal noise power in the receiver, are included in [1]-[7]. Initially, in [1] Tan and Beaulieu obtained an infinite representation series for the cumulative density function (CDF) of the bivariate Nakagami- $m$ distribution. In [2], Simon and Alouini obtained the outage probability and the average probability of error of dual selection combiners over correlated Nakagami- $m$ channels in integral forms using first-order Marcum $Q$-functions. In [3], Ko et al. analyzed the average SNR at the output of dual selection combiners over correlated Nakagami- $m$ fading. Recently, in [4] Tellambura et al. derived an expression of the moment generation function (MGF) in a dual selection combiner over correlated Nakagami- $m$ fading to obtain the average probability of error in coherent, differentially coherent, and noncoherent noise-limited systems. In [5], Ugweje analyzed performance metrics such as the probability density function (PDF), the average bit error rate and the outage probability of multiple SC over independent Nakagami- $m$ fading. The outage probability and the average probability of error of SC with multiple branches over correlated Nakagami- $m$ fading have been derived by Ugweje and Aalo in [6] using the multivariate gamma distribution. In [7], Zhang and Lu studied performance metrics of multiple SC over correlated Rayleigh, Rician and Rayleigh fading using the characteristic function. They analyzed the probability of error for differential phase shift keying (DPSK), noncoherent frequency shift keying (NCFSK), differential quadrature phase shift keying (DQPSK) with Gray coding and $M$-ary differential phase shift keying (M-DPSK) in this work.

The analysis of performance metrics in interference-limited scenarios (where the cochannel interference power prevails over the thermal noise) is addressed in [8]-[12]. The outage probability of dual SC over correlated Nakagami- $m$ fading was obtained in [8]-[10]. A signal-to-interference ratio (SIR) algorithm of selection was used in [8]-[9] whereas a desired power selection strategy was employed in [10]. In [11], Abu-Dayya and Beaulieu obtained outage probabilities over independent Nakagami- $m$ fading using 
desired signal power, total power, and SIR algorithms. Finally, in [12] Yao and Sheikh derived the outage probability of a multiple SC assuming total independence between signals and interferences on each branch of the combiner. Nevertheless, to the best of the authors' knowledge, there are no expressions available in the open technical literature for the performance metrics of dual SC over correlated Nakagami- $m$ fading with arbitrary fading parameters.

In this letter, the outage probability, the probability density function, the average signal-to-noise at the output of the combiner, and the probability of error of dual SC for binary modulations over correlated Nakagami- $m$ channels are derived assuming arbitrary fading parameters of signals at each branch of the combiner.

\section{NOISE-LIMITED SCENARIO}

In a noise-limited scenario, the outage probability, the average signal-to-noise ratio at the output of the combiner, and the probability of error are calculated only for thermal-noise, where the cochannel interference is negligible.

\section{A.- Outage probability}

We consider that the amplitude of the received signal on each branch of the combiner, which is denoted as $r_{j}(j=1,2)$, follows a Nakagami- $m$ distribution, where $m_{j}$ and $\Omega_{j}$ $(j=1,2)$ are the fading parameter and the average power, respectively.

From $[13,(15)]$ using the transformation $\varepsilon_{1}=r_{1}^{2}$ and $\varepsilon_{2}=r_{2}^{2}$, the joint CDF of the instantaneous power can be expressed as

$$
\begin{aligned}
F_{\varepsilon_{1}, \varepsilon_{2}}\left(\varepsilon_{1}, \varepsilon_{2}\right)= & \frac{(1-r)^{m_{2}}}{\Gamma\left(m_{1}\right)} \sum_{k=0}^{\infty} r^{k} \frac{\gamma\left(m_{1}+k, A \varepsilon_{1}\right)}{k !} \sum_{l=0}^{\infty} \frac{\Gamma\left(m_{2}-m_{1}+l\right)}{\Gamma\left(m_{2}-m_{1}\right) \Gamma\left(m_{2}+k+l\right) l !} r^{l}, \\
& \times \gamma\left(m_{2}+k+l, B \varepsilon_{2}\right), \quad \varepsilon_{1}, \varepsilon_{2} \geq 0 \quad 0 \leq r<1
\end{aligned}
$$

where $\Gamma(\cdot)$ denotes the gamma function $[14,(6.1 .1)] ; \gamma(\cdot, \cdot)$ is the incomplete gamma function $[14,(6.5 .2)] ; r$ is given by $r=\rho \sqrt{\frac{m_{2}}{m_{1}}}$ where $\rho$ is the correlation coefficient in power terms subjected to $m_{2} \geq m_{1}$; and

$$
\begin{aligned}
& A=\frac{m_{1}}{(1-r) \Omega_{1}}, \\
& B=\frac{m_{2}}{(1-r) \Omega_{2}} .
\end{aligned}
$$


The CDF of the maximum of two correlated random variables, $\varepsilon_{1}$ and $\varepsilon_{2}$, can be calculated as

$$
F_{\varepsilon}(\varepsilon)=\operatorname{Prob}\left(\varepsilon_{1}<\varepsilon, \varepsilon_{2}<\varepsilon\right)=\int_{0}^{\varepsilon} \int_{0}^{\varepsilon} p_{\varepsilon_{1}, \varepsilon_{2}}\left(\varepsilon_{1}, \varepsilon_{2}\right) d \varepsilon_{1} d \varepsilon_{2}=F_{\varepsilon_{1}, \varepsilon_{2}}(\varepsilon, \varepsilon), \quad \varepsilon \geq 0 .
$$

Let us define $s=\varepsilon E_{S} / N_{0}$ as the instantaneous SNR per symbol at the output of a SC, where $E_{S}$ is the energy per symbol and $N_{0}$ is the power spectral density. The average SNRs per symbol at each combiner input are given by $\bar{s}_{1}=\Omega_{1} E_{S} / N_{0}$ and $\bar{s}_{2}=\Omega_{2} E_{S} / N_{0}$, respectively. Thus, the probability of outage can be written as

$$
P_{\text {out }}(q)=\operatorname{Prob}(s<q)=F_{s}(q),
$$

where $q$ is the protection ratio of the SNR that is related to the required threshold probability of error as follows

$$
q=\left\{\begin{array}{cl}
\frac{1}{a} \ln \left(\frac{1}{2 p_{e}^{*}}\right) & \left\{\begin{array}{l}
a=\frac{1}{2} \text { for NCFSK } \\
a=1 \text { for DCPSK }
\end{array}\right. \\
\frac{1}{2 a}\left(Q^{-1}\left(p_{e}^{*}\right)\right)^{2} & \left\{\begin{array}{l}
a=\frac{1}{2} \text { for CFSK } \\
a=1 \text { for CPSK }
\end{array}\right.
\end{array}\right.
$$

where NCFSK and DCPSK apply to noncoherent frequency shift keying and differentially coherent phase shift keying, respectively; CFSK and CPSK represent coherent frequency shift keying and coherent phase shift keying, respectively; $Q^{-1}(\cdot)$ is the inverse of the normal probability integral; and $p_{e}^{*}$ is the required threshold probability of error. Note that (6) is applied to optimum detection with an optimum matched filter receiver [15].

From (5) and (6), the CDF of $s$ is obtained as

$$
\begin{aligned}
F_{s}(s)= & \frac{(1-r)^{m_{2}}}{\Gamma\left(m_{1}\right)} \sum_{k=0}^{\infty} r^{k} \frac{\gamma\left(m_{1}+k, C s\right)}{k !} \sum_{l=0}^{\infty} \frac{\Gamma\left(m_{2}-m_{1}+l\right)}{\Gamma\left(m_{2}-m_{1}\right) \Gamma\left(m_{2}+k+l\right) l !}, \\
& \times r^{l} \gamma\left(m_{2}+k+l, D s\right), \quad \mathrm{s} \geq 0 \quad 0 \leq r<1
\end{aligned}
$$

where

$$
C=\frac{m_{1}}{(1-r) \overline{s_{1}}}
$$

and

$$
D=\frac{m_{2}}{(1-r) \overline{s_{2}}}
$$


We have evaluated (7) with $n$ terms given by the Mathematica ${ }^{\circledR}$ Sum function. If the difference between the result of CDF for $n+1$ terms and the evaluation of CDF for $n$ is less than a specified accuracy, $n+1$ number of terms of summation is assumed for a specific accuracy. The number of terms required in (7) depends strongly on $s$ and $\rho$. Furthermore, the number of terms for a specific accuracy increases as $\rho$ and $s$ increase as occurs in the bivariate Nakagami- $m$ PDF infinite series [1, (Table I)]. For instance, the number of terms in the first and the second summation for $10^{-7}$ accuracy and the evaluation parameters $\left(m_{1}=1.2, m_{2}=1.5, \bar{s}_{1}=\bar{s}_{2}=1, r=0.11, \rho=0.1\right.$ and $\left.s=10\right)$ is 7. Nevertheless, if $r$ is increased to $0.89(\rho=0.8)$, the number of terms required for the same accuracy is 66 .

Fig. 1 shows the outage probability versus the average SNR at each branch of the combiner assuming $\bar{s}_{1}=\bar{s}_{2}$ for NCFSK and $p_{e}^{*}=10^{-4}$. The fading parameters of the signals at each input of the combiner are $m_{1}=1.2$ and $m_{2}=1.5$, respectively. The outage probability curves in a non-diversity environment with $m=1.2$ and $m=1.5$ have been plotted with solid lines. Dashed lines show the results for $\rho=0,0.3$ and 0.7. Significant performance differences exist between independent environments ( $\rho=$ $0)$ and high correlated environments $(\rho=0.7)$.

\section{B.- PDF of SNR}

The probability density function can be obtained from (7) using [14, (6.5.25)] as follows

$$
\begin{aligned}
p_{s}(s)= & \frac{(1-r)^{m_{2}}}{\Gamma\left(m_{2}-m_{1}\right) \Gamma\left(m_{1}\right)} \sum_{k=0}^{\infty} \sum_{l=0}^{\infty} r^{k+l} \frac{\Gamma\left(m_{2}-m_{1}+l\right)}{k ! l ! \Gamma\left(m_{2}+k+l\right)} \\
& \times\left[D^{m_{2}+k+l} s^{m_{2}+k+l-1} \exp (-D s) \gamma\left(m_{1}+k, C s\right)\right. \\
& \left.+C^{m_{1}+k} s^{m_{1}+k-1} \exp (-C s) \gamma\left(m_{2}+k+l, D s\right)\right], s \geq 0
\end{aligned}
$$

C.- Mean of the SNR

The mean of the SNR at the output of the combiner is calculated as

$$
\bar{s}=\int_{0}^{\infty} s p(s) d s .
$$

Using $[16,(6.455 / 2)]$, the integral in (11) can be evaluated as follows 


$$
\begin{aligned}
& \bar{s}=\frac{(1-r)^{m_{2}+1}}{\Gamma\left(m_{2}-m_{1}\right) \Gamma\left(m_{1}\right)} \sum_{k=0}^{\infty} \sum_{l=0}^{\infty} r^{k+l} \frac{\Gamma\left(m_{2}-m_{1}+l\right) \Gamma\left(m_{1}+m_{2}+2 k+l+1\right)}{k ! l ! \Gamma\left(m_{2}+k+l\right)} \\
& \times \frac{C^{m_{1}+k} D^{m_{2}+k+l}}{(C+D)^{m_{1}+m_{2}+2 k+l+1}}\left[\frac{1}{\left(m_{1}+k\right)}{ }_{2} F_{1}\left(1, m_{1}+m_{2}+2 k+l+1 ; m_{1}+k+1 ; \frac{1}{1+\frac{D}{C}}\right),\right. \\
& \left.+\frac{1}{\left(m_{2}+k+l\right)}{ }_{2} F_{1}\left(1, m_{1}+m_{2}+2 k+l+1 ; m_{2}+k+l+1 ; \frac{1}{1+\frac{C}{D}}\right)\right]
\end{aligned}
$$

where ${ }_{2} F_{1}(\cdot, \cdot ; \cdot ; \cdot)$ is the Gauss hypergeometric function [14, (15.1.1)].

The number of terms for a relative error, defined as $\left(\bar{s}_{n+1}-\bar{s}_{n}\right) / \bar{s}_{n+1}$, where $\bar{s}_{n}$ corresponds to (12) with a double summation limit of $n$, depends strongly on the correlation coefficient $\rho$. For instance, if $m_{1}=1.2, m_{2}=1.5, \bar{s}_{1}=\bar{s}_{2}=1, \rho=0.8$ and the relative error is fixed to $10^{-3}$, the number of terms required is 48 , whereas this number is reduced to 7 for $\rho=0.3$.

Fig. 2 shows the average signal-to-noise ratio at the output of the combiner versus the correlation coefficient between signals at each input of the combiner, assuming $\left(\bar{s}_{1}+\bar{s}_{2}\right) / 2=1$. The curves are plotted for $\bar{s}_{1}=\bar{s}_{2}=1 ; \bar{s}_{1}=1.5, \bar{s}_{2}=\bar{s}_{1} / 3=0.5$; and $\bar{s}_{1}=1.666, \bar{s}_{2}=\bar{s}_{1} / 5=0.333$. Higher unbalanced average SNRs at inputs of the combiner provide greater average SNRs at the output of the combiner.

D.- Average probability of error

The average probability of error can be obtained as

$$
\bar{P}_{e}=\int_{0}^{\infty} P_{e}(s) p_{s}(s) d s,
$$

where $P_{e}(s)$ is the probability of error given by

$$
P_{e}(s)= \begin{cases}\frac{1}{2} \exp (-a s) & \left\{\begin{array}{l}
a=\frac{1}{2} \text { for NCFSK } \\
a=1 \text { for DCPSK }
\end{array}\right. \\
Q(\sqrt{2 a s}) & \left\{\begin{array}{l}
a=\frac{1}{2} \text { for CFSK } \\
a=1 \text { for CPSK }
\end{array}\right.\end{cases}
$$

and $Q(\cdot)$ is the normal probability integral.

For NCFSK or DCPSK detection the average error probability can be calculated by substituting (10) and (14) in (13) using again [16, (6.455/2)] to give 


$$
\begin{aligned}
& \overline{P_{e}}=\frac{(1-r)^{m_{2}}}{\Gamma\left(m_{2}-m_{1}\right) \Gamma\left(m_{1}\right)} \sum_{k=0}^{\infty} \sum_{l=0}^{\infty} r^{k+l} \frac{\Gamma\left(m_{2}-m_{1}+l\right) \Gamma\left(m_{1}+m_{2}+2 k+l\right)}{k ! l ! \Gamma\left(m_{2}+k+l\right)} \\
& \times \frac{C^{m_{1}+k} D^{m_{2}+k+l}}{2(C+D+a)^{m_{1}+m_{2}+2 k+l}} \\
& \times\left[\frac{1}{\left(m_{1}+k\right)}{ }_{2} F_{1}\left(1, m_{1}+m_{2}+2 k+l ; m_{1}+k+1 ; \frac{C}{C+D+a}\right)\right. \\
& \left.+\frac{1}{\left(m_{2}+k+l\right)}{ }_{2} F_{1}\left(1, m_{1}+m_{2}+2 k+l ; m_{2}+k+l+1 ; \frac{C}{C+D+a}\right)\right]
\end{aligned}
$$

Again, the number of terms for a specific accuracy depends strongly on the correlation coefficient $\rho$. For instance, the number of terms for an accuracy of $10^{-7}$ in the evaluation of the average probability of error using NCFSK modulation is 43 for $m_{1}=1.2, m_{2}=$ $1.5, \bar{s}_{1}=\bar{s}_{2}=1$ and $\rho=0.8$. This number is reduced to 12 for $\rho=0.3$ with the same parameters.

The average probability of error for coherent detection (CFSK and CPSK) can be derived from (10), (13) and (14) as

$$
\begin{aligned}
\overline{P_{e}}= & \frac{(1-r)^{m_{2}}}{\Gamma\left(m_{2}-m_{1}\right) \Gamma\left(m_{1}\right)} \sum_{k=0}^{\infty} \sum_{l=0}^{\infty} r^{k+l} \frac{\Gamma\left(m_{2}-m_{1}+l\right)}{k ! l ! \Gamma\left(m_{2}+k+l\right)} \\
& \times \int_{0}^{\infty}\left[D^{m_{2}+k+l} s^{m_{2}+k+l-1} \exp (-D s) \gamma\left(m_{1}+k, C s\right) Q(\sqrt{2 a s}) .\right. \\
& \left.+C^{m_{1}+k} s^{m_{1}+k-1} \exp (-C s) \gamma\left(m_{2}+k+l, D s\right) Q(\sqrt{2 a s})\right] d s
\end{aligned}
$$

Using [16, (8.351/2), (7.621/4), (9.212/1)], [17, (06.27.26.0001.01)], it yields

$$
\begin{aligned}
& \bar{P}_{e}=\frac{(1-r)^{m_{2}}}{\Gamma\left(m_{2}-m_{1}\right) \Gamma\left(m_{1}\right)} \sum_{k=0}^{\infty} \sum_{l=0}^{\infty} r^{k+l} \frac{\Gamma\left(m_{2}-m_{1}+l\right)}{k ! l ! \Gamma\left(m_{2}+k+l\right)} C^{m_{1}+k} D^{m_{2}+k+l} \\
& \times\left[\frac{\Gamma\left(m_{1}+m_{2}+2 k+l\right)}{2\left(m_{1}+k\right)}{ }_{2} F_{1}\left(1, m_{1}+m_{2}+2 k+l ; m_{1}+k+1 ; \frac{C}{C+D}\right)\right. \\
& +\frac{\Gamma\left(m_{1}+m_{2}+2 k+l\right)}{2\left(m_{2}+k+l\right)}{ }_{2} F_{1}\left(1, m_{1}+m_{2}+2 k+l ; m_{2}+k+l+1 ; \frac{D}{C+D}\right) \\
& -\sqrt{\frac{a}{\pi} \Gamma\left(m_{1}+m_{2}+2 k+l+1 / 2\right)(C+D+a)^{-m_{1}-m_{2}-2 k-l-1 / 2}} \\
& \times\left(\frac{1}{m_{1}+k} F_{2}\left(m_{1}+m_{2}+2 k+l+1 / 2 ; 1,1 ; m_{1}+k+1,3 / 2 ; \frac{C}{C+D+a},-\frac{a}{C+D+a}\right)\right. \\
& \left.\left.-\frac{1}{m_{2}+k+l} F_{2}\left(m_{1}+m_{2}+2 k+l+1 / 2 ; 1,1 ; m_{2}+k+l+1,3 / 2 ; \frac{C}{C+D+a},-\frac{a}{C+D+a}\right)\right]\right)
\end{aligned}
$$

where $F_{2}(\cdot ; \cdot, \cdot ; \cdot, \cdot ; \cdot, \cdot)$ is the Appell hypergeometric function of the second kind given by $[16,(9.180 / 2)]$. The number of series terms for an accuracy of $10^{-7}\left(m_{1}=1.7, m_{2}=\right.$ 
$2.1, \bar{s}_{1}=\bar{s}_{2}=10$ and $\left.\rho=0.3\right)$ is 15 , whereas this number is increased to 52 for $\rho=0.7$ with the same value for the rest of parameters.

Fig. 3 shows the average error probability versus the average signal-to-noise ratio at each input of the combiner for NCFSK, assuming $\bar{s}_{1}=\bar{s}_{2}, m_{1}=1.2$, and $m_{2}=1.5$. Curves with dashed lines are depicted for $\rho=0,0.3$, and 0.7 .

\section{INTERFERENCE-LIMITED SCENARIO}

In interference-limited systems, thermal noise power is negligible compared to the cochannel interference power contribution.

\section{A.- Outage probability}

Expanding the confluent hypergeometric function in $[13,(12)]$ and using the transformation $\varepsilon_{1}=r_{1}^{2}$ and $\varepsilon_{2}=r_{2}^{2}$, the bivariate gamma PDF of the short-term power can be written as

$$
\begin{aligned}
& p_{\varepsilon_{1}, \varepsilon_{2}}\left(\varepsilon_{1}, \varepsilon_{2}\right)=\frac{(1-r)^{m_{2}}}{\Gamma\left(m_{1}\right)} \sum_{k=0}^{\infty} \sum_{l=0}^{\infty} \frac{\Gamma\left(m_{2}-m_{1}+l\right)}{\Gamma\left(m_{2}+k+l\right) \Gamma\left(m_{2}-m_{1}\right) k ! l !} r^{k+l} A^{m_{1}+k} B^{m_{2}+k+l}, \\
& \times \varepsilon_{1}^{m_{1}+k-1} \varepsilon_{2}^{m_{2}+k+l-1} \exp \left(-A \varepsilon_{1}\right) \exp \left(-B \varepsilon_{2}\right), \quad \varepsilon_{1}, \varepsilon_{2} \geq 0 \quad 0 \leq r<1
\end{aligned}
$$

where $A$ and $B$ are given by (2) and (3), respectively.

Let $\gamma_{1}$ and $\gamma_{2}$ be the signal-to-interference ratios at each input of the combiner calculated as

$$
\gamma_{j}=\frac{\varepsilon_{j}}{i_{j}}, \quad j=1,2
$$

where $i_{j}$ is the interference power at $j$ th input of the combiner.

The joint PDF of $\gamma_{1}$ and $\gamma_{2}$, assuming independence between $i_{1}$ and $i_{2}$, can be obtained as

$$
p_{\gamma_{1}, \gamma_{2}}\left(\gamma_{1}, \gamma_{2}\right)=\int_{0}^{\infty} \int_{0}^{\infty} i_{1} i_{2} p_{i_{1}}\left(i_{1}\right) p_{i_{2}}\left(i_{2}\right) p_{\varepsilon_{1}, \varepsilon_{2}}\left(i_{1} \gamma_{1}, i_{2} \gamma_{2}\right) d i_{1} d i_{2}
$$

where $p_{i_{1}}\left(i_{1}\right)$ and $p_{i_{2}}\left(i_{2}\right)$ are the PDFs of the total interference power received at each branch of the combiner.

Next, we consider that the total interference envelope is Nakagami- $m$ distributed where $m_{e}$ and $\Omega_{e}$ are the fading parameter and the average power of the interference amplitude, respectively. Thus, substituting (18) in (20) and using [16, (3.381/4)], the joint PDF of $\gamma_{1}$ and $\gamma_{2}$ can be derived as 


$$
\begin{aligned}
p_{\gamma_{1}, \gamma_{2}}\left(\gamma_{1}, \gamma_{2}\right)= & \frac{(1-r)^{m_{2}}}{\Gamma\left(m_{1}\right) \Gamma^{2}\left(m_{e}\right)} \sum_{k=0}^{\infty} \sum_{l=0}^{\infty} \frac{\Gamma\left(m_{2}-m_{1}+l\right) \Gamma\left(m_{1}+m_{e}+k\right) \Gamma\left(m_{2}+m_{e}+k+l\right)}{\Gamma\left(m_{2}+k+l\right) \Gamma\left(m_{2}-m_{1}\right) k ! l !} \\
& \times r^{k+l}\left(\frac{m_{e}}{\Omega_{e}}\right)^{m_{e}+k} \\
& \times \frac{\gamma_{1}^{m_{1}+k-1}}{\left(\frac{m_{e}}{\Omega_{e}}+A \gamma_{1}\right)^{m_{e}+m_{1}+k}} \frac{\gamma_{2}^{m_{2}+k+l-1}}{\left(\frac{m_{e}}{\Omega_{e}}+B \gamma_{2}\right)^{m_{e}+m_{2}+k+l}}, \quad \gamma_{1}, \gamma_{2} \geq 0 \quad 0 \leq r<1
\end{aligned}
$$

The CDF of the maximum signal-to-interference ratio of $\gamma_{1}$ and $\gamma_{2}$, can be calculated as

$$
F_{\gamma}(\gamma)=\int_{0}^{\gamma} \int_{0}^{\gamma} p_{\gamma_{1}, \gamma_{2}}\left(\gamma_{1}, \gamma_{2}\right) d \gamma_{1} d \gamma_{2}
$$

The outage probability, which is defined as the probability of the instantaneous SIR at the output of the combiner is less than the SIR protection ratio, $q_{I}$, can be obtained from (21) and (22) using [16,(3.197/1)] as

$$
\begin{aligned}
P_{\text {out }}\left(q_{I}\right)= & F_{\gamma}\left(q_{I}\right)=\frac{(1-r)^{m_{2}}}{\Gamma\left(m_{1}\right) \Gamma^{2}\left(m_{e}\right)} \sum_{k=0}^{\infty} \sum_{l=0}^{\infty} \frac{\Gamma\left(m_{2}-m_{1}+l\right) \Gamma\left(m_{1}+m_{e}+k\right)}{\left(m_{1}+k\right)\left(m_{2}+k+l\right) \Gamma\left(m_{2}+k+l\right)} \\
& \times \frac{\Gamma\left(m_{2}+m_{e}+k+l\right)}{\Gamma\left(m_{2}-m_{1}\right) k ! l !} r^{k+l}\left(\frac{\Omega_{e}}{m_{e}}\right)^{m_{1}+m_{2}+2 k+l} \quad A^{m_{1}+k} B^{m_{2}+k+l} q_{I}^{m_{1}+m_{2}+2 k+l} \\
& \times{ }_{2} F_{1}\left(m_{1}+k, m_{1}+m_{e}+k ; m_{1}+k+1 ;-\frac{A \Omega_{e} q_{I}}{m_{e}}\right) \\
& \times{ }_{2} F_{1}\left(m_{2}+k+l, m_{2}+m_{e}+k+l ; m_{2}+k+l+1 ;-\frac{B \Omega_{e} q_{I}}{m_{e}}\right), \quad 0 \leq r<1
\end{aligned}
$$

For $m_{1}=m_{2}=m,(23)$ agrees with $[8,(16)]$ assuming independence between interferences that are received on both branches of the combiner.

In Fig. 4, the outage probability versus the average signal-to-interference ratio $\left(S I R_{a v}\right)$ that is normalized with the protection ratio, $q_{I}$, is plotted for $m_{1}=1.2$ and $m_{2}=1.5$, assuming $\Omega_{1}=\Omega_{2}$, where $S I R_{a v}$ is defined as

$$
S I R_{a v}=\frac{\Omega}{\Omega_{e}}=\frac{\left(\frac{\Omega_{1}+\Omega_{2}}{2}\right)}{\Omega_{e}} .
$$

The outage probability is represented by curves with dashed lines for correlation coefficients $\rho=0,0.3$ and 0.7 . 


\section{CONCLUSIONS}

In this letter, performance metrics of dual selection combiners over correlated Nakagami- $m$ fading have been derived analytically assuming arbitrary fading parameters of signals and arbitrary average powers at each input of the combiner. The expression series for the average probability of error, the average signal-to-noise ratio at the combiner output, and the outage probability have also been obtained. Our performance results for high correlation coefficients differ substantially from those obtained by other authors for total independence between signals at each input of the combiner. We consider that the results of performance metrics obtained in previous works, assuming total independence between fading signals are excessively optimistic. Our results cover more realistic scenarios, where fading parameters on each branch of the combiner are arbitrary.

\section{ACKNOWLEDGMENT}

The authors would like to thank the anonymous reviewers and the Editor for their helpful comments which considerably improved the quality of this letter. 


\section{REFERENCES}

[1] C. C. Tan and N. C. Beaulieu, "Infinite series representation of the bivariate Rayleigh and Nakagami-m distributions," IEEE Trans. Commun., vol. 45, no. 10, pp. 1159-1161, Oct. 1997.

[2] M. K. Simon and M.-S. Alouini, "A unified performance analysis of digital communication with dual selective combining diversity over correlated Rayleigh and Nakagami fading channels," IEEE Trans. Commun., vol. 47, no. 1, pp. 3343, Jan. 1999.

[3] Y-C. Ko, M.-S. Alouini, and M. K. Simon, "Average SNR of dual selection combining over correlated Nakagami- $m$ fading channels," IEEE Commun. Lett., vol. 4, no. 1, pp. 12-14, Jan. 2000.

[4] C. Tellambura, A. Annamalai and V.K. Bhargava, "Closed form and infinite series solutions for the MGF of a dual-diversity selection combiner output in bivariate Nakagami fading," IEEE Trans. Commun., vol. 51, no. 4, pp. 539-542, Apr. 2003.

[5] O. C. Ugweje, "Selection diversity for Wireless Communications in Nakagamifading with arbitrary parameters," IEEE Trans. Veh. Technol., vol. 50, no. 6, pp. 1437-1448, Nov. 2001.

[6] O. C. Ugweje and V. A. Aalo, "Performance of selection diversity system in correlated Nakagami fading," Proc. IEEE Veh. Technol. Conference Spring. Phoenix AZ, May, 1997. pp. 1488-1492.

[7] Q. T. Zhang and H. G. Lu, "A general analytical approach to multi-branch selection combining over various spatially correlated fading channels," IEEE Trans. Commun., vol. 50, no. 7, pp. 1066-1073, Jul. 2002.

[8] J. Reig, N. Cardona and L. Rubio, "Outage probabilities of dual selection combiners over correlated Nakagami fading with multiple interferers," in Proc. Wireless Personal Multimedia Commun. Aalborg, Denmark, 2001.

[9] G. K. Karagiannidis, "Performance analysis of SIR-based dual selection diversity over correlated Nakagami-m fading channels," IEEE Trans. Veh. Technol., vol. 52, no. 5, pp. 1207-1216, Sept 2003.

[10] J. Reig, N. Cardona and L. Rubio, "Outage probabilities of dual selection combiners in a correlated Nakagami fading with cochannel interference," in Proc. IEEE Veh. Technol. Conference Spring. Rodes, Greece, 2001. pp. 1488-1492. 
[11] A. A. Abu-Dayya and N.C. Beaulieu, "Outage probabilities of diversity cellular systems with cochannel interference in Nakagami fading," IEEE Trans. Veh. Technol., vol. 41, no. 4, pp. 343-355, Nov. 1992.

[12] Y. D. Yao and A. U. H. Sheikh, "Investigations into cochannel interference in microcellular mobile radio systems," IEEE Trans. Veh. Technol., vol. 41, no. 2, pp. 114-123, May. 1992.

[13] J. Reig, N. Cardona, and L. Rubio, "Bivariate Nakagami-m distribution with arbitrary fading parameters," Electronics Letters, vol. 38, no. 25, pp. 1715-1717, Dec. 2002.

[14] M. Abramowitz and I. A. Stegun, Handbook of Mathematical Functions. New York: Dover, 1972.

[15] M. Schwartz, W. R. Bennett and S. Stein, Communication Systems and Techniques, New York: McGraw-Hill, 1966.

[16] I. S. Gradsthteyn and Ryzhik, I.M., Table of Integrals, Series and Products, 5-th ed. New York: Academic Press, Inc., 1994.

[17] Wolfram. (2005) The Wolfram functions site. Internet. [Online]. Available: http://functions.wolfram.com. 